\title{
A Method for Identification and Assessment of Radioxenon Plumes by Absorption in Polycarbonates
}

\author{
Dobromir Pressyanov*(D) and Pavel Stavrev (D) \\ Faculty of Physics, University of Sofia "St. Kliment Ohridski", 5 James Bourchier Blvd., 1164 Sofia, Bulgaria; \\ pstavrev@phys.uni-sofia.bg \\ * Correspondence: pressyan@phys.uni-sofia.bg
}

Citation: Pressyanov, D.; Stavrev, P. A Method for Identification and Assessment of Radioxenon Plumes by Absorption in Polycarbonates. Sensors 2021, 21, 8107. https://doi.org/ $10.3390 / \mathrm{s} 21238107$

Academic Editor: Heinrich J. Wörtche

Received: 27 September 2021

Accepted: 30 November 2021

Published: 3 December 2021

Publisher's Note: MDPI stays neutral with regard to jurisdictional claims in published maps and institutional affiliations.

Copyright: (c) 2021 by the authors. Licensee MDPI, Basel, Switzerland. This article is an open access article distributed under the terms and conditions of the Creative Commons Attribution (CC BY) license (https:/ / creativecommons.org/licenses/by/ $4.0 /)$.

\begin{abstract}
A method for the retrospective evaluation of the integrated activity concentration of ${ }^{133} \mathrm{Xe}$ during radioxenon plumes and the moment of the plume's center is proposed and explored by computer modeling. The concept is to use a specimen of polycarbonate material (a stack of Makrofol $\mathrm{N}$ foils of thickness $120 \mu \mathrm{m}$ and $40 \mu \mathrm{m}$ in $1 \mathrm{~L}$ non-hermetic Marinelly beaker) that is placed in the environment or in a controlled nuclear or radiopharmaceutical facility. On a regular basis or incidentally, the specimen may be retrieved and gamma spectrometry in two consecutive time intervals with durations of $8 \mathrm{~h}$ and $16 \mathrm{~h}$ is performed. To assess the performance of the method, ${ }^{133} \mathrm{Xe}$ plumes of various integrated activity concentrations and with a duration of up to $10 \mathrm{~h}$ are simulated and analyzed, assuming that the measurement starts with a delay of up to one day after the moment of the plume center. It is found that the deviation between the estimates by the method and their true values are within a few percent. Depending on the delay, events of integrated ${ }^{133} \mathrm{Xe}$ activity concentration $250-1000 \mathrm{~Bq} \mathrm{~h} \mathrm{~m}^{-3}$ might be qualitatively identified. At levels $>10,000 \mathrm{~Bq} \mathrm{~h} \mathrm{~m}^{-3}$, the uncertainty of the quantitative estimates might be $\leq 10 \%$.
\end{abstract}

Keywords: radioxenon plumes; ${ }^{133} \mathrm{Xe}$; absorption in polycarbonates; Makrofol; retrospective estimates; nuclear accidents

\section{Introduction}

Among the man-made radioactive noble gases, the isotopes of xenon (radioxenon, isotopes of interest are ${ }^{135} \mathrm{Xe},{ }^{133} \mathrm{Xe},{ }^{133 \mathrm{~m}} \mathrm{Xe}$ and ${ }^{131 \mathrm{~m}} \mathrm{Xe}$ ) attract particular attention. These isotopes are released from nuclear and radiopharmaceutical facilities and from hospitals, where ${ }^{133} \mathrm{Xe}$ is administered to patients. They are among the key radionuclides whose release from nuclear power plants (NPPs) has to be monitored [1]. In the event of a nuclear emergency, most of their inventory in the nuclear installation may be released into the environment [2]. Radioxenon plays a key role in the control of the Treaty on the NonProliferation of Nuclear Weapons and the Comprehensive Test Ban Treaty (CTBT) and a world monitoring network has been set up for this purpose [3]. In the environment, the concentrations of ${ }^{133} \mathrm{Xe}$ usually exceed those of other xenon isotopes by several orders of magnitude [4], but, after a subsurface nuclear explosion, the ratio of ${ }^{135} \mathrm{Xe} /{ }^{133} \mathrm{Xe}$ may be four orders of magnitude larger compared, for instance, to a reactor release [5]. The estimated release of ${ }^{133} \mathrm{Xe}$ from NPPs in North America and Europe ranges within $10^{7}-10^{11} \mathrm{~Bq} /$ day per one NPP unit [6], mostly as continuous emissions. The emissions from radiopharmaceutical factories are substantially higher [7] and, usually, these are "pulse" short-term releases, reaching activity of $10^{15} \mathrm{~Bq}$ in several hours, in some cases [4]. The ${ }^{133}$ Xe release after the Chernobil accident was estimated to be between $1.33 \times 10^{17}$ and $6.5 \times 10^{18} \mathrm{~Bq}[8]$ and the ${ }^{133}$ Xe release after the Fukushima Daiichi accident in 2011 was estimated to be of the order of $10^{19} \mathrm{~Bq}$ [9]. For comparison, after a $1 \mathrm{kt}$ nuclear explosion of plutonium A-bomb, approximately $10^{15} \mathrm{~Bq}$ of ${ }^{133} \mathrm{Xe}$ will be emitted within three hours of the explosion $[10,11]$. 
Continuous reactor releases are hardly detectable at long distances from NPPs. In contrast, radioxenon plumes with a duration of several hours and integrated over the duration of the plume ${ }^{133} \mathrm{Xe}$ activity concentration of more than $1 \mathrm{kBq} \mathrm{h} \mathrm{m}{ }^{-3}$ may be observed tens of kilometers away from the radiopharmaceutical facilities after short-term releases of ${ }^{133} \mathrm{Xe}$ of the order of $10^{13}-10^{14} \mathrm{~Bq}$ [4]. At such distances, the plumes after a major nuclear emergency can be expected to have an integrated activity concentration that is 3-5 orders of magnitude greater. They are also detected at much longer distances: after the Fukushima Daiichi accident, ${ }^{133} \mathrm{Xe}$ activity concentrations of up to $30-70 \mathrm{~Bq} \mathrm{~m}{ }^{-3}$ were detected at distances $>6500 \mathrm{~km}$ from the site of emergency $[12,13]$. The plumes at longer distances also last longer due to the plume dispersion during atmospheric transport.

Measuring ${ }^{133} \mathrm{Xe}$ in the environment is a challenge. Thus far, there are no compact and mobile monitors that are sufficiently sensitive to be used for measurement in the environment around nuclear and radiopharmaceutical facilities. The sensitivity of the stationary monitors used in nuclear installation is not sufficient for environmental control, and, in the case of a major nuclear emergency, they may become inoperative. The radioxenon detection systems used in CTBT stations are highly sensitive, but, after a major nuclear emergency, they may reach their upper limit of detection and become inoperative. On the other hand, strategic decisions rely on information about ${ }^{133} \mathrm{Xe}$ in the environment, especially after a nuclear emergency, accidental release from nuclear and radiopharmaceutical facilities or in the case of possible clandestine nuclear actions.

The use of the high absorption ability of noble gases by bisphenol-A based polycarbonates (BPA-PCs) [14] for ${ }^{222} \mathrm{Rn}$ measurements was first proposed in 1999 [15] and for the measurements of ${ }^{85} \mathrm{Kr}$ and ${ }^{133} \mathrm{Xe}$ in 2004 [16]. Since then, many studies have focused on the further development of this "polycarbonate method" for natural [17] and man-made radioactive noble gases [18-21]. In particular, different BPA-PCs have been studied and Makrofol $\mathrm{N}^{\circledR}$ (Bayer AG) has been identified as the material that has the highest absorption ability for noble gases [22-24]. Thus far, the potential of this method for ${ }^{133}$ Xe measurement has been studied for long-term (days/weeks) continuous exposures [19,21]. However, accidental radioxenon releases, as well as those from radiopharmaceutical facilities, are mostly short-term "pulses", which are registered in the close environment as radioxenon plumes with a duration of several hours [4].

In this work, we propose a method to identify, qualitatively, ${ }^{133}$ Xe plumes and to evaluate, quantitatively, the integrated ${ }^{133} \mathrm{Xe}$ activity concentration and the moment of the plume center. The method consists of placing in the environment or within the controlled facility of polycarbonate specimens formed as a stack of Makrofol $\mathrm{N}$ foils of two different thicknesses, which are placed in a non-hermetic canister. The specimens can be left for a long time and taken for analysis only in the case of suspected high radioxenon release or on a regular basis, e.g., daily, for performing regular control. Then, the specimens are transported to a laboratory, which may be distant from the point of exposure, and measured via HPGe gamma spectrometry, using the $81 \mathrm{keV}$ line of ${ }^{133} \mathrm{Xe}$. By measuring the signal (net area of the $81 \mathrm{keV}$ peak spectral region of interest) in two different time intervals, the integrated activity concentration of ${ }^{133} \mathrm{Xe}$ during the plume and the time (before the start of the measurement) of the "center of the plume" can be evaluated. By computer modeling of realistic situations, it is demonstrated that the method provides feasible results and is sufficiently sensitive in the range that is frequently encountered in the environment, e.g., around radiopharmaceutical factories [4]. The specimen can be analyzed up to one day after the plume has dissipated.

\section{Materials and Methods}

\subsection{The Concept and Basics of the Method}

The decay scheme and the basic nuclear data of the isotope ${ }^{133} \mathrm{Xe}$ are shown in Figure 1 [25]. 
${ }^{133}$ Xe Half-life $=5.2474(5) \mathrm{d}$

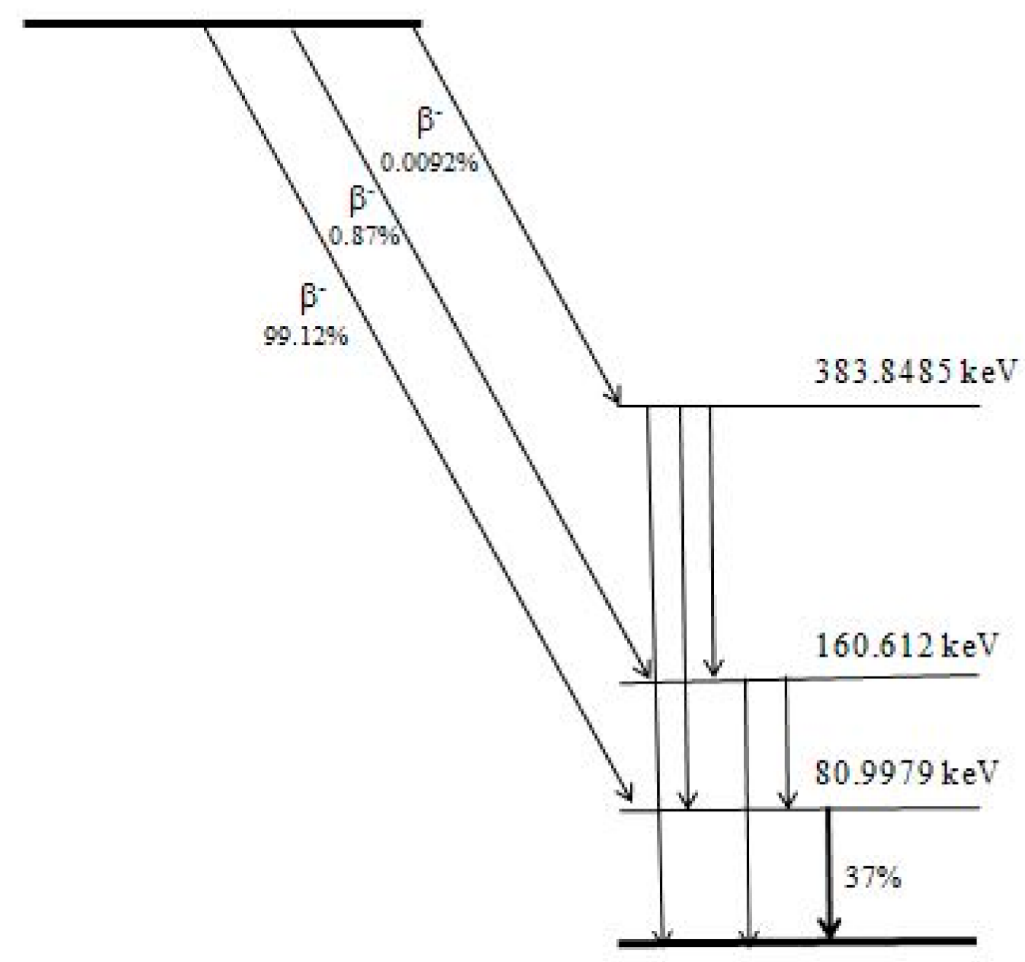

${ }^{133} \mathrm{Cs}$

Figure 1. Decay scheme of ${ }^{133} \mathrm{Xe}$. The half-life, probability of the decay modes and energy of the excited states are shown, using the data of Ref. [25]. The present method uses the gamma-line of energy $80.9972(\approx 81 \mathrm{kev})$ that is of emission probability of $37 \%$.

Here, we employ the concept of a "composite absorber", consisting of a stack of absorbing foils. When the foils are not hermetically stuck together, the noble gas may penetrate freely between them. Initially, this concept was proposed and tested for radon measurement [22,26-29] and further evaluated for ${ }^{133} \mathrm{Xe}$, too [19]. Previous experiments with ${ }^{222} \mathrm{Rn}$ have shown good agreement between the theoretical modeling of such "composite absorbers" and experimental results [29]. For simulation purposes, the following experimental setup is assumed, namely Makrofol $\mathrm{N}$ foils of thickness $40 \mu \mathrm{m}$ and $120 \mu \mathrm{m}$, stuck together and placed in a canister, specifically a $1 \mathrm{~L}$ Marinelli beaker, as shown in Figure 2. Half of the volume of the beaker is filled with $40 \mu \mathrm{m}$ and half of it with $120 \mu \mathrm{m}$, and the foils of each type can be considered evenly distributed within the volume.

The transport of a radioactive isotope of a noble gas in a polycarbonate is described by the diffusion equation with radioactive decay $[18,21,30]$. For the geometry of thin foils of thickness $L$, it is given by Equation (1) [30]:

$$
\frac{\partial n}{\partial t}=D \frac{\partial^{2} n}{\partial x^{2}}-\lambda n
$$

where $n$ is the atomic concentration of the noble gas in the polycarbonate material, $x=0$ and $x=L$ are the coordinates of the foil boundaries, $D$ is the diffusion coefficient of xenon in the polycarbonate material, and $\lambda$ is the decay constant of the isotope (in the present case, this is $\left.{ }^{133} \mathrm{Xe}\right)$. During exposure, the initial and boundary conditions are $n(x, t=0)=0$ and $n(x=0, t)=n(x=L, t)=K n_{0}(t)$, where $n_{0}$ is the atom concentration of the noble gas in the ambient air and $K$ is the "partition coefficient" (dimensionless solubility) of the gas in the material ( $K=$ ratio of the concentration in an "infinitely" thin surface layer of the material to that in the air). After exposure, the boundary conditions are zero and the initial 
distribution of $n$ within the foil is that at the end of the preceding exposure. This model was found to perfectly fit the experimental data $[18,21,24,30]$.

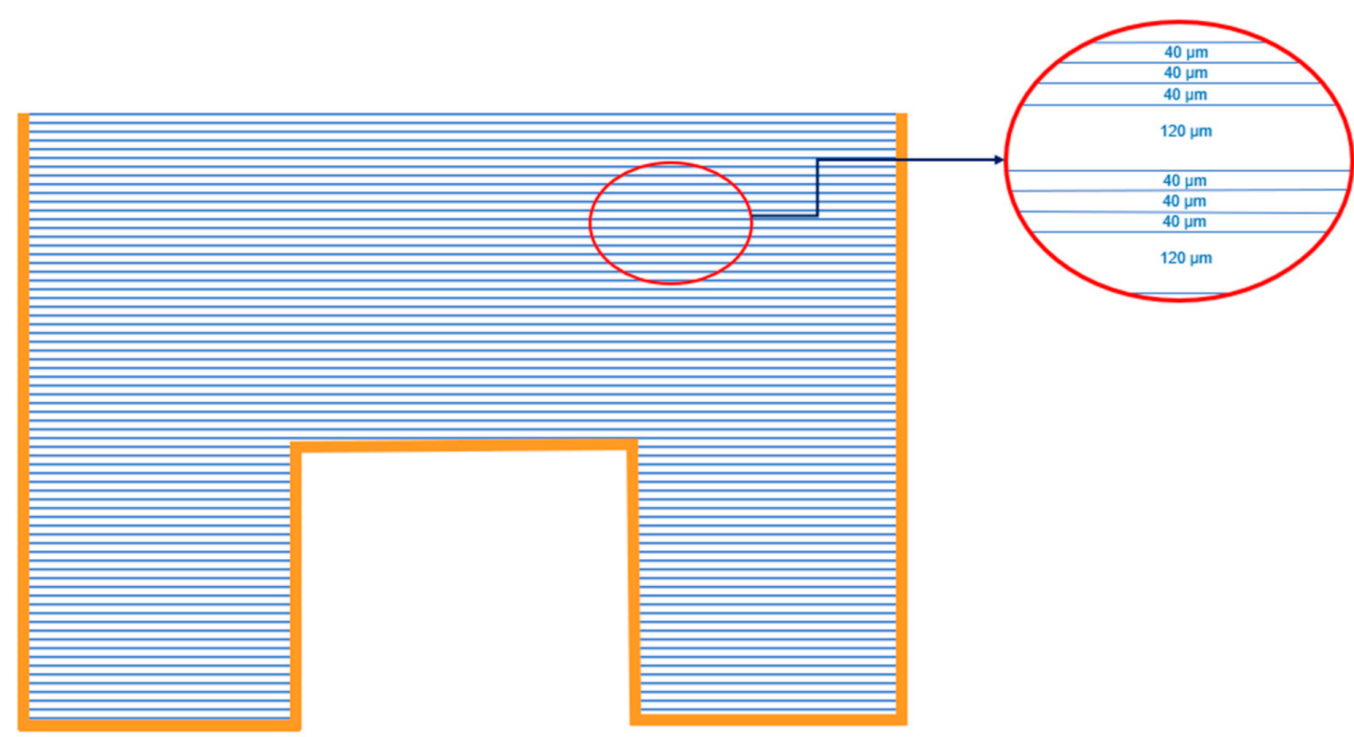

Figure 2. Placement of Makrofol $\mathrm{N}$ foils in $1 \mathrm{~L}$ Marinelli beaker for exposure in the environment. Above one $120 \mu \mathrm{m}$ foil, three $40 \mu \mathrm{m}$ foils are placed and this is repeated until the volume is filled.

The exact solutions of Equation (1) have been obtained elsewhere [18,30]. According to these solutions, during exposure to a constant activity concentration $c_{A}$ in air $\left(c_{A}=\lambda n_{0}\right)$, the growth in activity in a stack of foils of thickness $L$ and of total volume $V$ can be expressed as:

$$
A(t)=\int_{V} \lambda n(x, t) d V=c_{A} V\left\{\frac{8 \lambda K L_{D}^{2}}{L^{2}} \sum_{k=0}^{\infty} \frac{1-\exp \left(-\lambda_{2 k+1} t\right)}{\lambda_{2 k+1}}\right\}
$$

where $L_{D}$ is the diffusion length of the considered isotope in the material $\left(L_{D}=\sqrt{D / \lambda}\right)$ and the constants $\lambda_{2 \mathrm{k}+1}$ are determined as follows:

$$
\lambda_{2 k+1}=\lambda\left(1+\left((2 k+1) \pi \frac{L_{D}}{L}\right)^{2}\right)
$$

Here, values of $K=17.2$ and $L_{D}\left({ }^{133} \mathrm{Xe}\right)=87.8 \mu \mathrm{m}$ were used, according to data in Ref. [24]. In Ref. [24], $L_{D}$ was reported for ${ }^{131 \mathrm{~m}} \mathrm{Xe}$, and, as the diffusion coefficient for all Xe isotopes is the same, it was recalculated for ${ }^{133} \mathrm{Xe}$ as follows:

$$
L_{D}\left({ }^{133} \mathrm{Xe}\right)=L_{D}\left({ }^{131 m} \mathrm{Xe}\right) \sqrt{\lambda\left({ }^{131 m} \mathrm{Xe}\right) / \lambda\left({ }^{133} \mathrm{Xe}\right)}
$$

After the end of exposure at moment $T$, the activity decreases due to the radioactive decay and outgazing. The process is described by expression (5) [30]:

$$
A(t)=c_{A} V\left\{\frac{8 \lambda K L_{D}^{2}}{L^{2}} \sum_{k=0}^{\infty} \frac{1-\exp \left(-\lambda_{2 k+1} T\right)}{\lambda_{2 k+1}} \exp \left(-\lambda_{2 k+1} t\right)\right\}
$$

where $t$ is the time after the end of exposure. Notably, the thinner the foil, the faster is the decrease in the activity [19].

Let $A_{1}(t)$ be the activity, according to Equation (5), of a volume $V$ filled with foils of $L=L_{1}$ (in the present case, $L_{1}=40 \mu \mathrm{m}$ ) and $A_{2}(t)$ is when it is filled with foils of 
$L=L_{2}\left(L_{2}=120 \mu \mathrm{m}\right)$. Then, the activity of the composite absorber of volume $V$, when $\frac{1}{2} V$ is filled with foils of thickness $L_{1}$ and another $\frac{1}{2} V$ with foils of $L_{2}$, is given by Equation (6):

$$
A(t)=\frac{1}{2}\left(A_{1}(t)+A_{2}(t)\right)=c_{A} V\left\{4 \lambda K\left[\frac{L_{D}^{2}}{L_{1}^{2}} \sum_{k=0}^{\infty} \frac{1-\exp \left(-\lambda_{2 k+1}^{(1)} T\right)}{\lambda_{2 k+1}^{(1)}} \exp \left(-\lambda_{2 k+1}^{(1)} t\right)+\frac{L_{D}^{2}}{L_{2}^{2}} \sum_{k=0}^{\infty} \frac{1-\exp \left(-\lambda_{2 k+1}^{(2)} T\right)}{\lambda_{2 k+1}^{(2)}} \exp \left(-\lambda_{2 k+1}^{(2)} t\right)\right]\right\}
$$

where the constants $\lambda_{2 k+1}^{(i)}$ correspond to foils of thickness $L_{i}(i=1,2)$. Figure 3 illustrates the time dependence of $A(t)$.

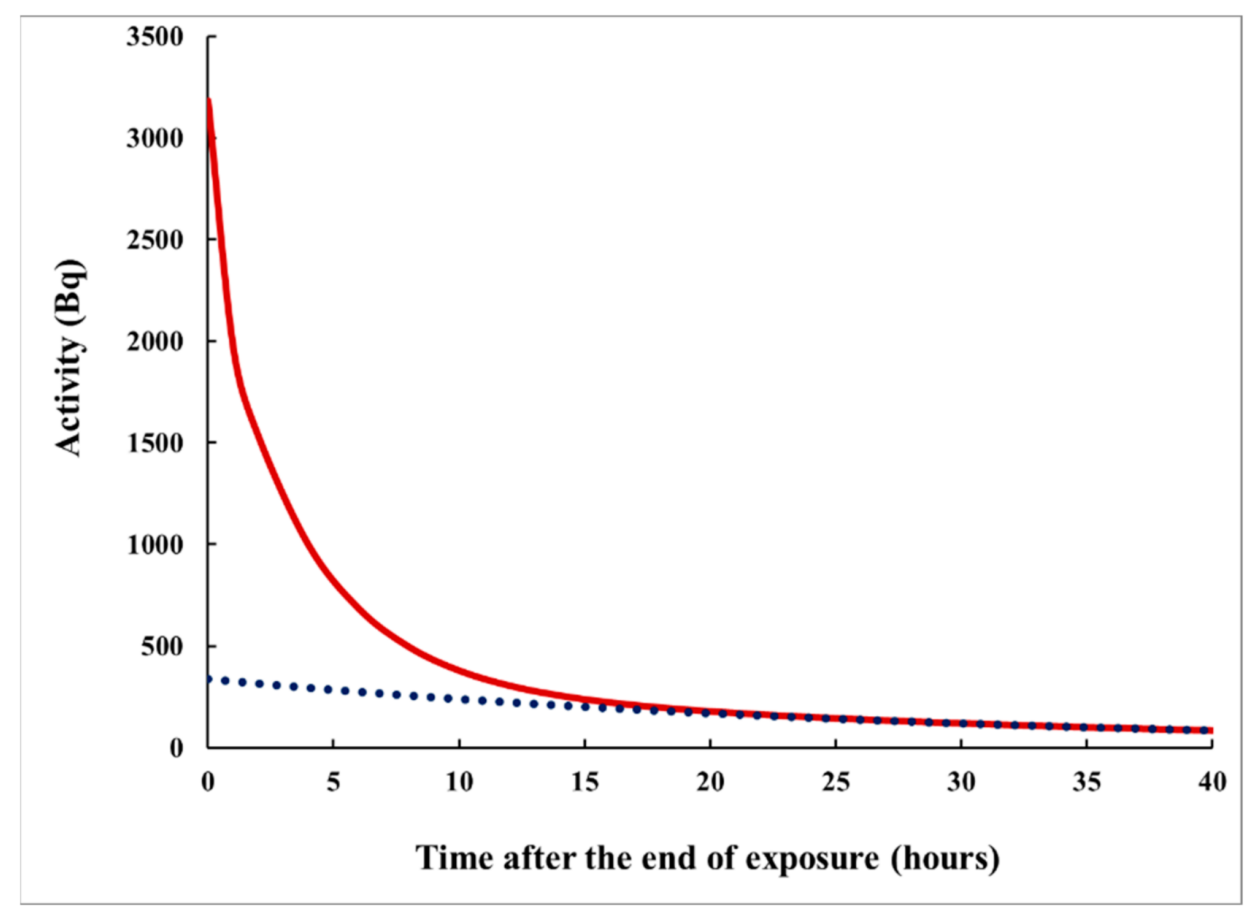

Figure 3. The decrease in the activity in the specimen (due to radioactive decay and outgazing) after the end of exposure, according to eqn. (6). The initial activity of the specimen (at the end of exposure) is $3200 \mathrm{~Bq}$. The dotted line corresponds to the "slowest component" in the sum (6), that is $\sim \exp \left(-\lambda_{1}^{(2)} T\right)$. Note that the ratio $A(\tau) / A(t+\tau)$ at fixed $t$ depends on $\tau$; therefore, $\tau$ might be correlated with $S_{1} / S_{2}$.

When using HPGe gamma spectrometry, the measured signal $(S)$ is the net-area of the $81 \mathrm{keV}$ peak region of interest (ROI). In data processing, the detection efficiency $(\varepsilon)$ and the probability $(p)$ for $81 \mathrm{keV}$ gamma emission in the ${ }^{133} \mathrm{Xe}$ decay should be taken into account. For modeling purposes, it is assumed that the specimens taken for analysis are measured by HPGe gamma spectrometry in two consecutive time intervals: the first of duration $t_{1}=8 \mathrm{~h}$ (signal $S_{1}$ ) and the second of duration $t_{2}=16 \mathrm{~h}\left(S_{2}\right)$-the duration of the whole measurement is $t_{1}+t_{2}=24 \mathrm{~h}$. In the general case, the time interval when the plume has occurred is not known and some time for transportation of the specimen to the measuring laboratory may be needed. Therefore, the measurement starts with some delay $\tau_{d}$ after the end of the plume (or $\tau$ after its "center", for rectangular plumes of duration $T$ : $\left.\tau=\tau_{d}+T / 2\right)$. Therefore, the modeled signals $S_{1}$ and $S_{2}$ in the first and the second interval are as follows:

$$
\begin{gathered}
S_{1}=\varepsilon p \int_{t_{d}}^{\tau_{d}+t_{1}} A(t) d t=\varepsilon p c_{A} V\left\{4 \lambda K \left[\frac{L_{D}^{2}}{L_{1}^{2}} \sum_{k=0}^{\infty} \frac{1-\exp \left(-\lambda_{2 k+1}^{(1)} T\right)}{\lambda_{2 k+1}^{(1)} \lambda_{2 k+1}^{(1)}} \exp \left(-\lambda_{2 k+1}^{(1)} \tau_{d}\right)\left[1-\exp \left(-\lambda_{2 k+1}^{(1)} t_{1}\right)\right]+\right.\right. \\
\left.\left.\frac{L_{D}^{2}}{L_{2}^{2}} \sum_{k=0}^{\infty} \frac{1-\exp \left(-\lambda_{2 k+1}^{(2)} T\right)}{\lambda_{2 k+1}^{(2)} \lambda_{2 k+1}^{(2)}} \exp \left(-\lambda_{2 k+1}^{(2)} \tau_{d}\right)\left[1-\exp \left(-\lambda_{2 k+1}^{(2)} t_{1}\right)\right]\right]\right\},
\end{gathered}
$$


where the activity $A(t)$ is that given by Equation (6). Respectively, for $S_{2}$, one obtains:

$$
\begin{aligned}
S_{2} & =\varepsilon p \int_{t_{d+t_{1}}}^{\tau_{d}+t_{1}+t_{2}} A(t) d t \\
& =\varepsilon p c_{A} V\left\{4 \lambda K \left[\frac{L_{D}^{2}}{L_{1}^{2}} \sum_{k=0}^{\infty} \frac{1-\exp \left(-\lambda_{2 k+1}^{(1)} T\right)}{\lambda_{2 k+1}^{(1)} \lambda_{2 k+1}^{(1)}} \exp \left(-\lambda_{2 k+1}^{(1)}\left(\tau_{d}+t_{1}\right)\right)\left[1-\exp \left(-\lambda_{2 k+1}^{(1)} t_{2}\right)\right]\right.\right. \\
& \left.\left.+\frac{L_{D}^{2}}{L_{2}^{2}} \sum_{k=0}^{\infty} \frac{1-\exp \left(-\lambda_{2 k+1}^{(2)} T\right)}{\lambda_{2 k+1}^{(2)} \lambda_{2 k+1}^{(2)}} \exp \left(-\lambda_{2 k+1}^{(2)}\left(\tau_{d}+t_{1}\right)\right)\left[1-\exp \left(-\lambda_{2 k+1}^{(2)} t_{2}\right)\right]\right]\right\}
\end{aligned}
$$

It could be seen in Figure 3 that, after sufficient delay (e.g., $>20 \mathrm{~h}$ ) following the end of exposure, the time dependence is determined mainly by one component of the sum (7): that with $\lambda_{1}^{(2)}$, which is the smallest constant in the set of $\lambda_{2 k+1}^{(1,2)}$ constants (in present case, $\left.\lambda_{1}^{(2)}=0.0346 \mathrm{~h}^{-1}\right)$. This component is also dominating in the times when $S_{2}$ is acquired $(t>8 \mathrm{~h})$. Therefore, at the times when this component dominates, the following approximation becomes useful:

$$
A(t) \approx \frac{L_{D}^{2}}{L_{2}^{2}} \frac{1-\exp \left(-\lambda_{1}^{(2)} T\right)}{\lambda_{1}^{(2)}} \exp \left(-\lambda_{1}^{(2)} t\right)
$$

The next approximation is for a plume duration of a few hours. With the quoted $\lambda_{1}{ }^{(2)}$, for such plumes, $\lambda_{1}{ }^{(2)} T$ is much smaller than one, and 1-exp $\left(-\lambda_{1}^{(2)} T\right) \approx \lambda_{1}^{(2)} T$, which, for $T=1 \mathrm{~h}$, is valid within $2 \%$; for $T=8 \mathrm{~h}$, within $8 \%$, and for $T=10 \mathrm{~h}$, within $15 \%$. Therefore,

$$
\frac{L_{D}^{2}}{L_{2}^{2}} \frac{1-\exp \left(-\lambda_{1}^{(2)} T\right)}{\lambda_{1}^{(2)}} \exp \left(-\lambda_{1}^{(2)} t\right) \approx \frac{L_{D}^{2}}{L_{2}^{2}} \operatorname{Texp}\left(-\lambda_{1}^{(2)} t\right)
$$

Using the approximations (7) and (8), one obtains, if the conditions of the approximations are met,

$$
S_{2} \approx c_{A} T\left\{\varepsilon p V \frac{4 \lambda K L_{D}^{2}}{L_{2}^{2}} \frac{\exp \left(-\lambda_{1}^{(2)}\left(\tau_{d}+t_{1}\right)\right)\left(1-\exp \left(-\lambda_{1}^{(2)} t_{2}\right)\right)}{\lambda_{1}^{(2)}}\right\}=I_{A}\left\{\varepsilon p V \frac{4 \lambda K L_{D}^{2}}{L_{2}^{2}} \frac{\exp \left(-\lambda_{1}^{(2)}\left(\tau_{d}+t_{1}\right)\right)\left(1-\exp \left(-\lambda_{1}^{(2)} t_{2}\right)\right)}{\lambda_{1}^{(2)}}\right\}
$$

where $I_{A}=c_{A 0} T$ is the time-integrated concentration of ${ }^{133} \mathrm{Xe}$ for the considered rectangular plume. In addition, for plumes of different shapes, the end of the plume is not a welldefined moment and, preferably, the delay $(\tau)$ should be measured from its center, as shown in Figure 4. The following "working hypothesis", raised for plumes of any shape and of any duration, so far not exceeding $10 \mathrm{~h}$, is at the core of the method:

- For measurement in two consecutive time intervals, (0-8 h) and (8-24 h), the observed ratio $S_{1} / S_{2}$ can be correlated with the delay $\tau$, measured from the center of the plume, and the dependence $\tau=\tau\left(S_{1} / S_{2}\right)$ can be used to assess the delay $\tau$ of a plume of any shape.

- $\quad$ The signal $S_{2}$ may be expressed as:

$$
S_{2}=\varepsilon p \frac{4 \lambda V K L_{D}^{2}}{L_{2}^{2}} \frac{I_{A}}{\lambda_{1}^{(2)}} \exp \left(-\lambda_{1}^{(2)}\left(\tau+t_{1}\right)\right)\left(1-\exp \left(-\lambda_{1}^{(2)} t_{2}\right)\right) g(\tau)
$$

where $g(\tau)$ is a "corrective function" that depends only on the delay $\tau$ and that accounts for the difference in (11) from the exact expression (8), which is due to the approximations made and to the replacing of $\tau_{d}$ with $\tau$ for the delay. 


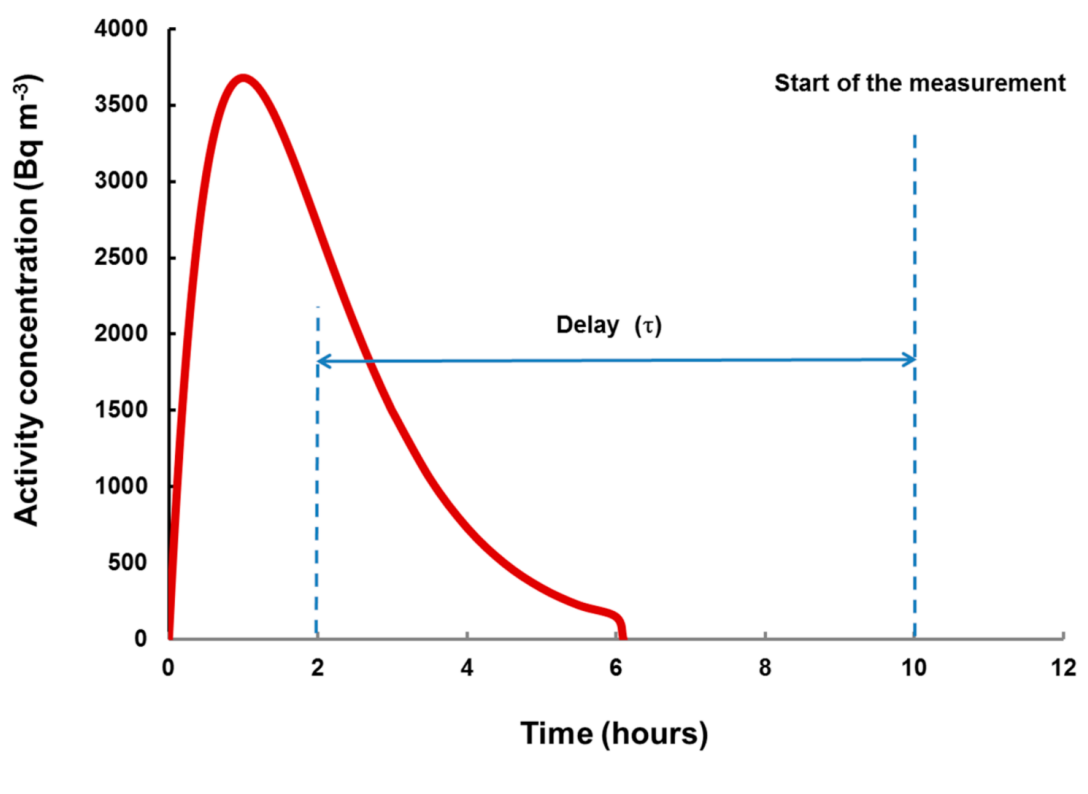

Figure 4. An arbitrary example of a plume. As the "end of plume" may be not well-defined, it is preferred to use the delay from the center of the plume. The moment of this center is $=\int t c_{A} d t / \int c_{A} d t$ (for rectangular plumes, it is $T / 2$ ).

The dependence $\tau\left(S_{1} / S_{2}\right)$ was obtained by simulation of rectangular plumes of different duration and different delay $\tau$, and looking for the best fit, which can be interpolated by analytical expression. Using the same simulation data, the corrective function $g(\tau)$ was modeled by the following expression, obtained from Equation (12):

$$
g(\tau)=\frac{S_{2}(\text { true })}{\varepsilon p \frac{4 \lambda V K L_{D}^{2}}{L_{2}^{2}} \frac{I_{A}}{\lambda_{1}^{(2)}} \exp \left(-\lambda_{1}^{(2)}\left(\tau+t_{1}\right)\right)\left(1-\exp \left(-\lambda_{1}^{(2)} t_{2}\right)\right)},
$$

where $S_{2}($ true) is that calculated by Equation (8).

When the above conditions are met and $\tau\left(S_{1} / S_{2}\right)$ and $g(\tau)$ are known, for any arbitrary plume, using the measured $S_{1}$ and $S_{2}$, the delay $\tau$ is determined from $\tau=\tau\left(S_{1} / S_{2}\right)$ and $I_{A}$ is determined as follows:

$$
I_{A}=\frac{1}{\varepsilon p} \frac{L_{2}^{2}}{L_{D}^{2}} \frac{S_{2} \lambda_{1}^{(2)}}{4 \lambda V K} \exp \left(\lambda_{1}^{(2)} \tau\right) \frac{1}{\left(\exp \left(-\lambda_{1}^{(2)} t_{1}\right)-\exp \left(-\lambda_{1}^{(2)}\left(t_{1}+t_{2}\right)\right)\right) g(\tau)}
$$

Computer modeling, based on the analytical expressions described in this paper, was carried out in order to prove that, based on this hypothesis, one may obtain reliable results for the delay $\tau$ and the integrated activity concentration $I_{A}$. For different $c_{A}, T$ and $\tau$, the signals $S_{1}$ and $S_{2}$ were calculated using the exact expressions (7) and (8). The correlation between $\tau$ and $S_{1} / S_{2}$ is illustrated in Figure 5 . The dependence is practically one and the same for rectangular plumes of duration $1-5 \mathrm{~h}$. 


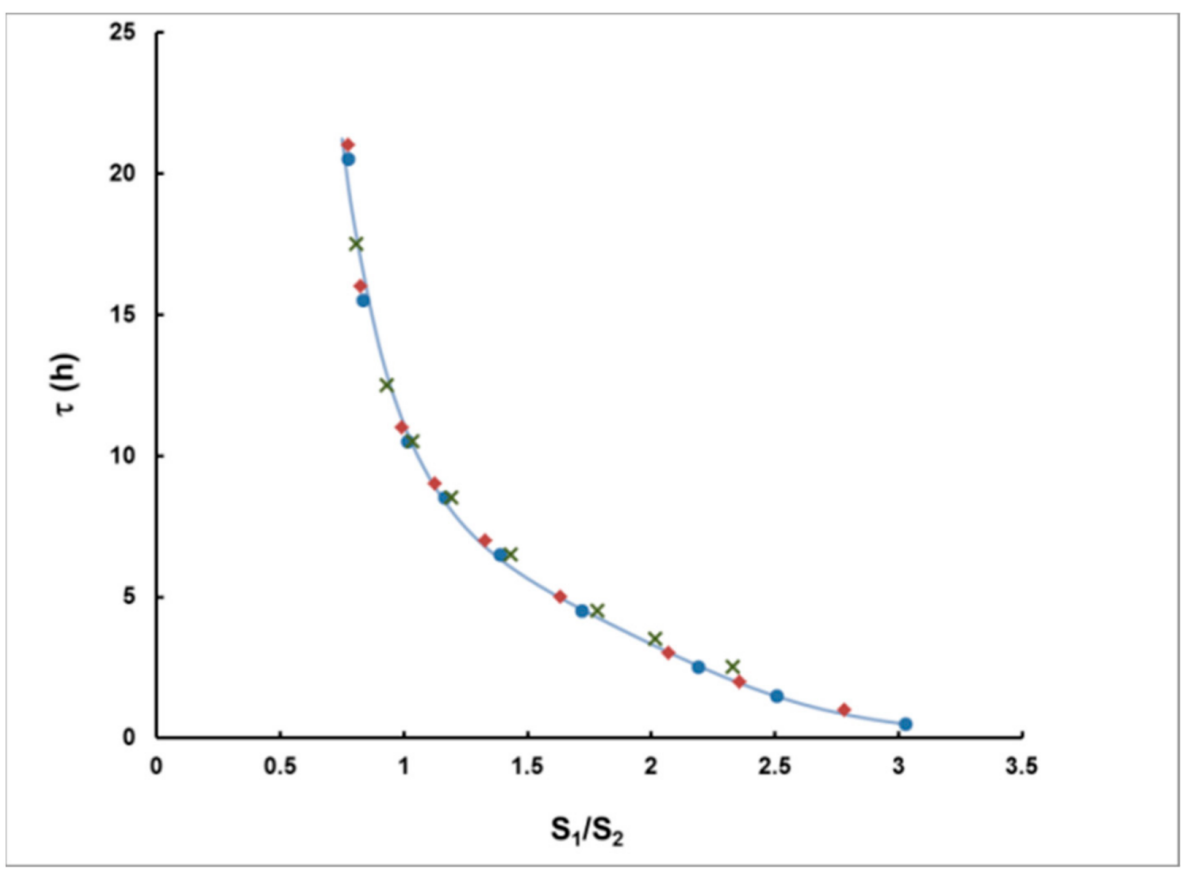

Figure 5. Delay $(\tau)$ as a function of $S_{1} / S_{2}$. Simulated rectangular plumes of duration $1 \mathrm{~h}(\bullet), 2 \mathrm{~h}(\diamond)$ and $5 \mathrm{~h}(\times)$ are fitted by the equation (15) (the solid curve).

The data are very well-interpolated by the following expression (the curve in Figure 5), which is hereafter used to determine $\tau$ from $S_{1} / S_{2}$ :

$$
\tau[h]=\exp \left(0.2987\left(\frac{S_{1}}{S_{2}}\right)^{4}-2.6469\left(\frac{S_{1}}{S_{2}}\right)^{3}+8.0991\left(\frac{S_{1}}{S_{2}}\right)^{2}-11.455\left(\frac{S_{1}}{S_{2}}\right)+8.1133\right),
$$

The results for $g$ are illustrated in Figure 6. As seen, the results depend on $\tau$, but practically not on $T$.

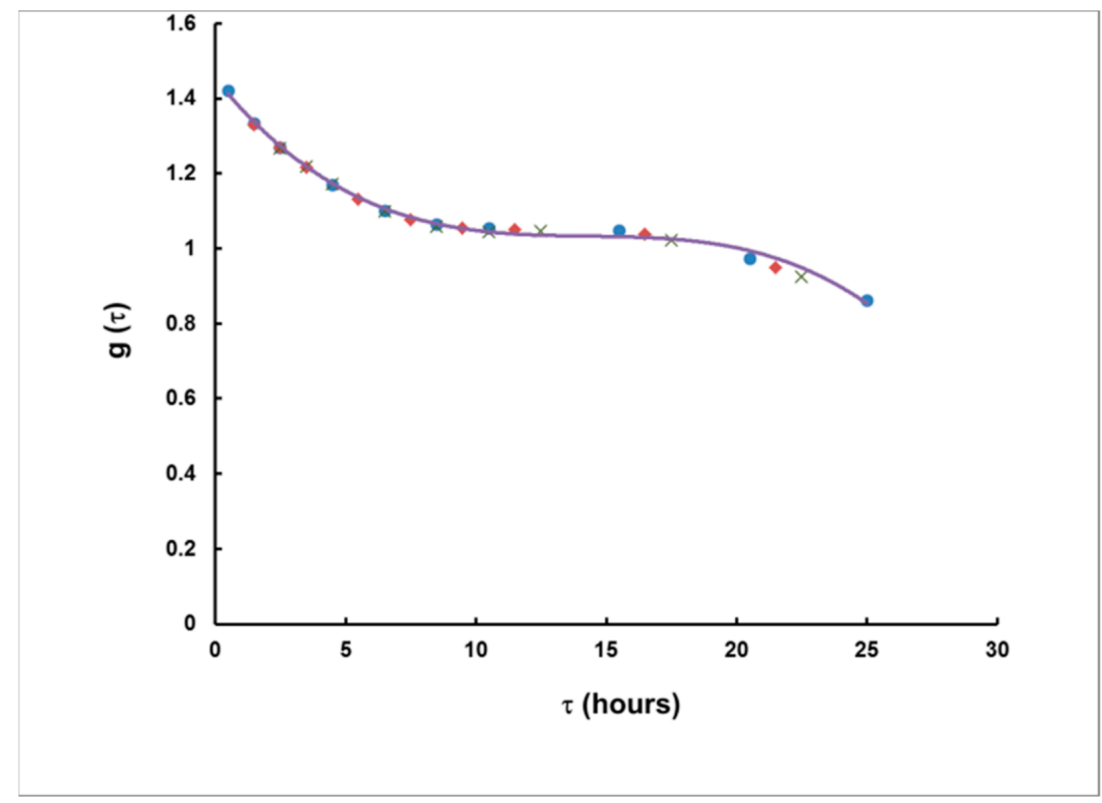

Figure 6. The corrective function $g$ as a function of $\tau$. Points correspond to rectangular plumes of duration $1 \mathrm{~h}(\bullet), 3 \mathrm{~h}(\diamond)$ and $5 \mathrm{~h}(\times)$. The solid curve represents a third-degree polynomial fit to the data points (Equation (14)). 
This dependence was very well-interpolated analytically, by the following polynomial:

$$
g(\tau)=-0.000138 \tau^{3}+0.005945 \tau^{2}-0.086074 \tau+1.453013,
$$

where $\tau$ is previously obtained by Equation (15). The obtained interpolations of $\tau\left(S_{1} / S_{2}\right)$ and $g(\tau)$ were used in the data processing of simulated plumes of any shape and of different delay.

\subsection{Estimating the Uncertainty and the Level of Plume Identification}

Both $I_{A}$ and $\tau$ are functions of $S_{1}$ and $S_{2}$, which are uncorrelated variables. In real measurements, they are random numbers of "counting uncertainty" $\sigma\left(S_{1}\right)$ and $\sigma\left(S_{2}\right)$, which can be determined by the Poisson distribution of the total number of counts and the background. Using the interpolation expressions for $\tau\left(S_{1} / S_{2}\right), g\left(\tau\left(S_{1} / S_{2}\right)\right)$ and replacing them in Equation (14), one obtains the function $I_{A}\left(S_{1}, S_{2}\right)$. Then, the uncertainties are calculated, using the common uncertainty propagation formulas for functions of uncorrelated variables [31]:

$$
\begin{gathered}
\sigma(\tau)=\sqrt{\left(\frac{\partial \tau}{\partial S_{1}}\right)^{2} \sigma^{2}\left(S_{1}\right)+\left(\frac{\partial \tau}{\partial S_{2}}\right)^{2} \sigma^{2}\left(S_{2}\right)} \\
\sigma\left(I_{A}\right)=\sqrt{\left(\frac{\partial I_{A}}{\partial S_{1}}\right)^{2} \sigma^{2}\left(S_{1}\right)+\left(\frac{\partial I_{A}}{\partial S_{2}}\right)^{2} \sigma^{2}\left(S_{2}\right)}
\end{gathered}
$$

The level of plume identification $I_{A}{ }^{(\min )}$ is the minimum level at which the signal will exceed the background at $95 \%$ probability. This problem has been considered elsewhere [32], where it has been shown that, for a "well-defined background" (assumed in our modeling), this is valid when the signal is:

$$
S>L_{c}=1.64 \sqrt{n_{b} t}
$$

where $n_{b}$ is the "blank counting rate" (in the case of gamma spectrometry, it will be continuum+background counting rate in the region of interest of the analytical peak) and $t$ is the duration of the measurement $\left(t=t_{1}\right.$ for $S_{1}$ and $t_{2}$ for $S_{2}$, respectively). Simulations were carried out to obtain the integrated activity concentration $I_{A}{ }^{(m i n)}$, which corresponds to the "critical level" $L_{c}$. Notably, this is a level of qualitative "identification" indicating the existence of an event that resulted in a ${ }^{133}$ Xe signal above the background at $95 \%$ statistical significance. To have reliable quantitative estimates of $\tau$ and $I_{A}$, the signal should be sufficiently above the "level of identification", as shown in the Results section.

\section{Results}

\subsection{Methodological Bias in the Estimates of the of Delay and the Integrated Concentration}

Figure 7 illustrates the agreement between the true and calculated time delay according to Equation (15). In this case, rectangular plumes with a duration of up to $10 \mathrm{~h}$ were considered. For time delays of up to $20 \mathrm{~h}$, a very good match was observed between the true and calculated time delay values. There was a systematic bias for time delays higher than $20 \mathrm{~h}$, which increased with the increase in the delay. Therefore, at this stage, the use of the proposed method should be restricted only to situations in which the measurement starts no later than $20 \mathrm{~h}$ after the moment of the "plume center". 


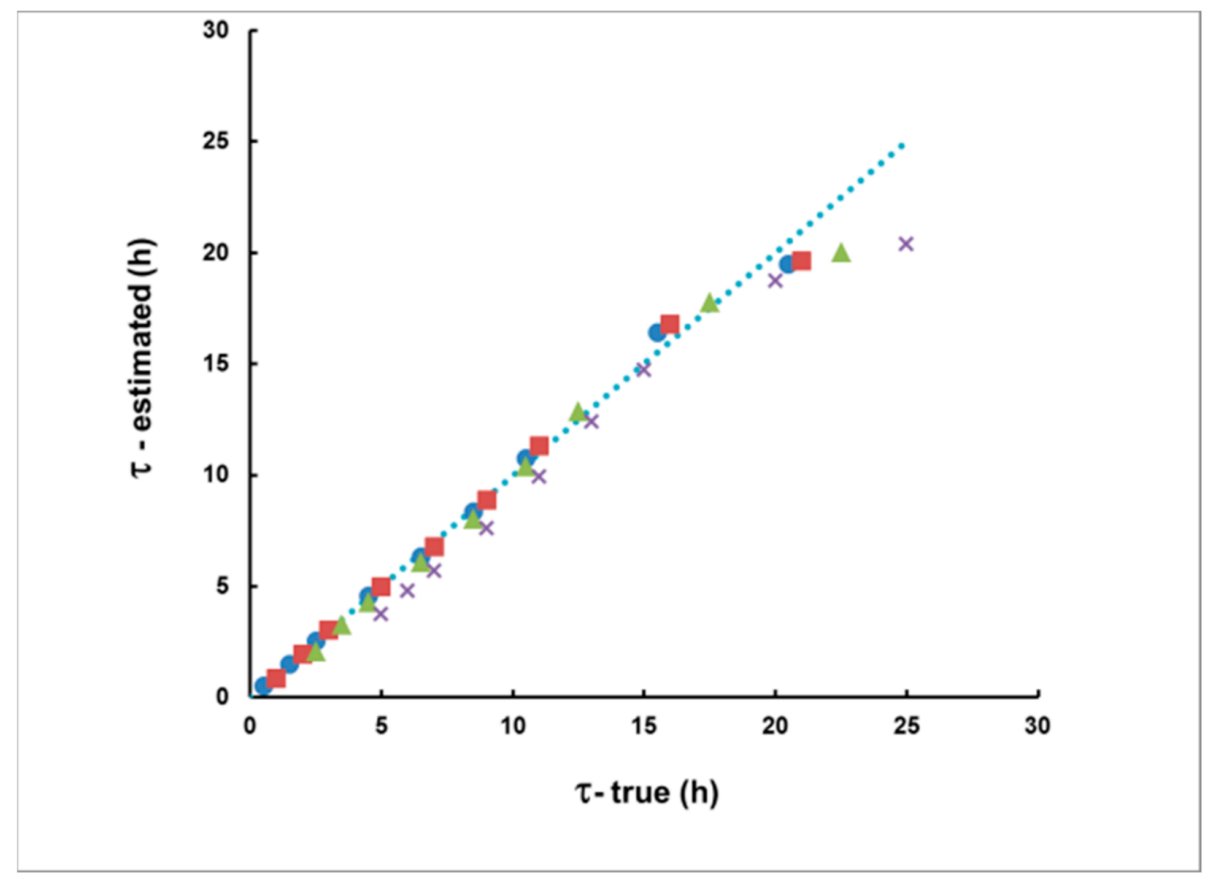

Figure 7. The estimated, according to $S_{1} / S_{2}$ delay, compared with the true delay of rectangular plumes of duration $1 \mathrm{~h}(\bullet), 2 \mathrm{~h}(\square), 5 \mathrm{~h}(\Delta)$ and $10 \mathrm{~h}(\times)$. The dotted line represents the coincidence between the estimated and true values.

Using Equation (14) and the estimated value of the delay time, one can calculate the values of $I_{A}$. Figure 8 illustrates the relative deviation of $I_{A}$ determined by Equation (16) from its true value for the case of rectangular plumes with a duration of up to $10 \mathrm{~h}$ and measurement started with a delay of up to $20 \mathrm{~h}$. As seen in the figure, all relative deviations fall within $6 \%$, while most of them fall within $2 \%$. In most real cases, such bias will be sufficiently smaller than the uncertainty incurred by counting statistics.

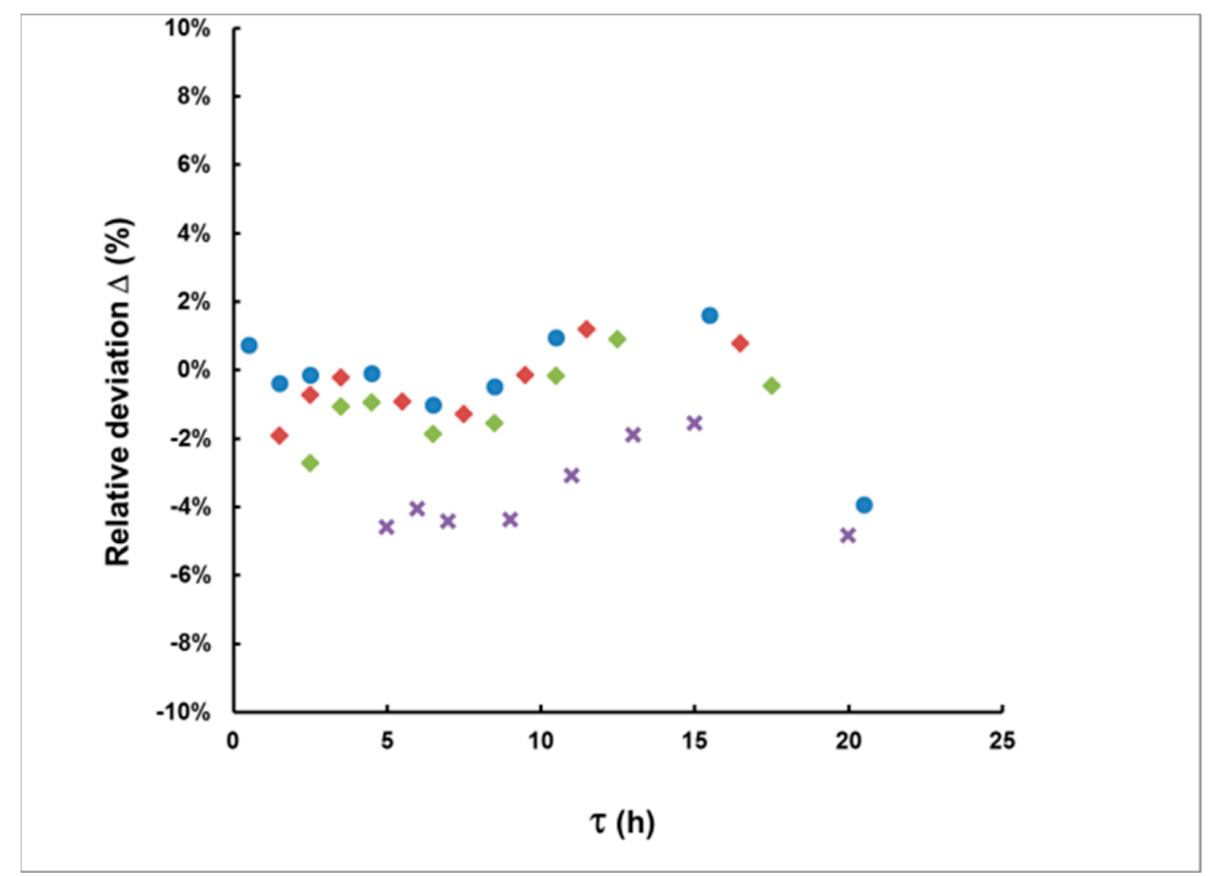

Figure 8. The relative deviation in $I_{A}\left(\Delta=\left(I_{A}\right.\right.$ (calculated) $-I_{A}$ (true) $) / I_{A}$ (true) $)$ for delays of up to $20 \mathrm{~h}$. The duration of the simulated rectangular plumes is $1 \mathrm{~h}(\bullet), 3 \mathrm{~h}(\diamond), 5 \mathrm{~h}(\diamond)$ and $10 \mathrm{~h}(\times)$. 


\subsection{Simulation of Levels of Identification and Instrumental Uncertainty}

The characteristics of the gamma spectrometer employed for research and education, in our laboratory, were used in the modeling. The spectrometer was supplied with a HPGe detector $\left(\right.$ ORTEC ${ }^{\circledR}$ ) of $24.9 \%$ relative efficiency and a resolution (FWHM) of $1.9 \mathrm{keV}$ for the $1332 \mathrm{keV}$ line of ${ }^{60} \mathrm{Co}$. Gamma spectrometry analysis uses the line of ${ }^{133} \mathrm{Xe}$ with energy $81 \mathrm{keV}$ ( $p=0.37$, see Figure 1) [25]. When the background (with a shielded detector) is measured for long time, the average counting rate in the region of interest of the $81 \mathrm{keV}$ peak is approximately $1 \mathrm{cpm}(60 \mathrm{imp} /$ hour $)$ and this value has been used in the modeling. Thus, the background counts in the first interval with a duration of $8 \mathrm{~h}$ will be $480(8 \times 60)$ and in the second interval with a duration of $16 \mathrm{~h}$ will be $960(16 \times 60)$ correspondingly.

By substituting this background, one may calculate the "critical levels" $L c_{1}, L c_{2}$, for the corresponding intervals (see Equation (19)). The values of $\mathrm{I}_{\mathrm{A}}$ that "generate" signals $S_{1}=L_{C 1}$ or $S_{2}=L c_{2}$ will be "the level for qualitative identification". This level depends on the choice of the intervals used for identification and on the time delay. The results for the level of identification after numerical simulations are illustrated in Figure 9 as dependent on the delay. As seen in this figure, if $S_{1}+S_{2}$ is used ( $24 \mathrm{~h}$ spectrum acquisition), a plume with an integrated activity concentration greater than $1000 \mathrm{~Bq} \mathrm{~h} \mathrm{~m}{ }^{-3}$ will lead to a signal that would exceed the background at a $95 \%$ level of confidence, and levels as low as $250 \mathrm{~Bq} \mathrm{~h} \mathrm{~m}{ }^{-3}$ may be qualitatively identified if the measurement starts with a delay $<2 \mathrm{~h}$. Even measurements within the first $8 \mathrm{~h}\left(\mathrm{~S}_{1}\right)$ may be conclusive for the identification of $<1000 \mathrm{~Bq} \mathrm{~h} \mathrm{~m}{ }^{-3}$, provided that the delay does not exceed $10 \mathrm{~h}$. This means that most of the radioxenon plumes observed, e.g., in Ref. [4], tens kilometers away from radiopharmaceutical facilities, would be identified.

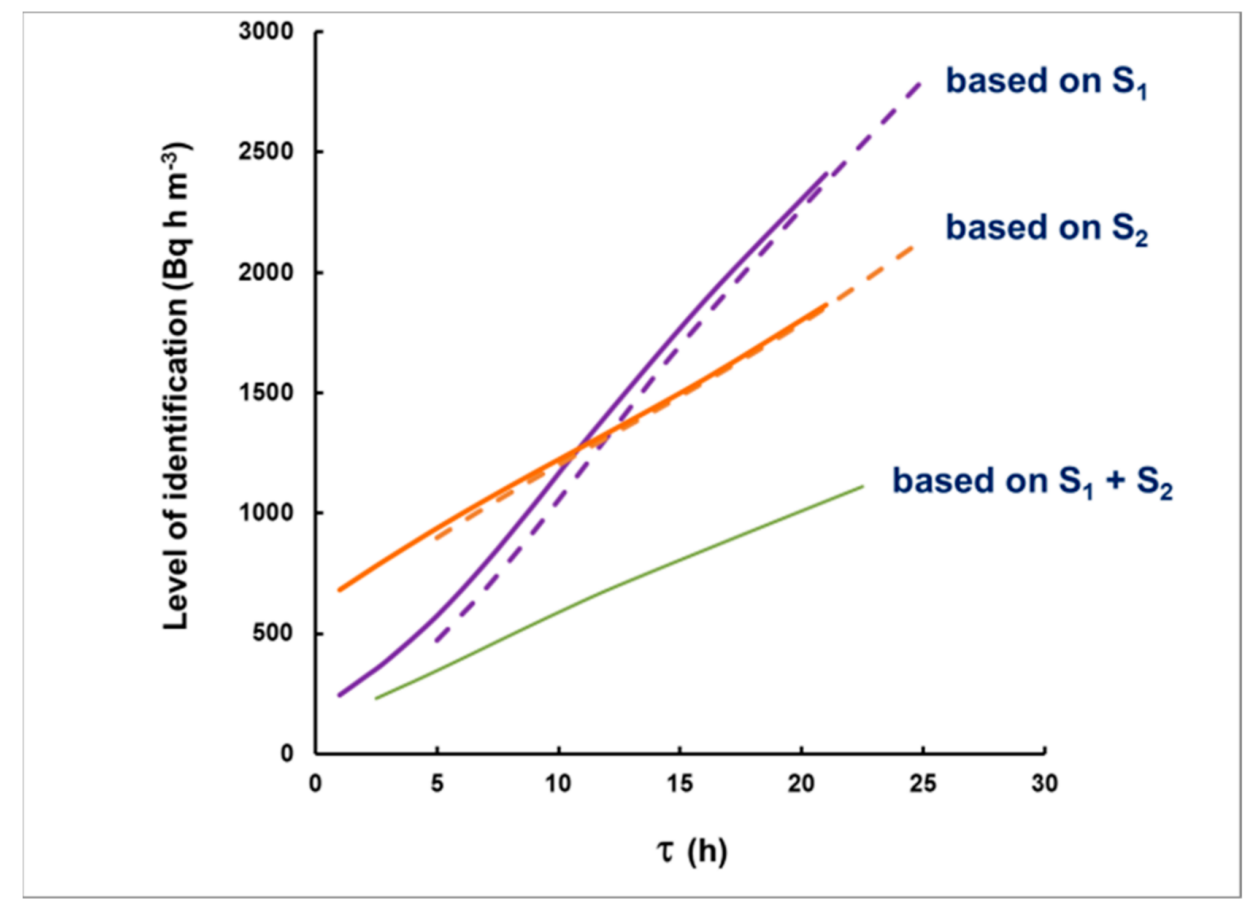

Figure 9. The dependence of the "level of identification" on the delay, when using $S_{1}$ (violet), $S_{2}$ (orange) or $S_{1}+S_{2}$ (green). Even for $S_{1}$, the dependence on plume duration is weak (dashed line). The solid lines are for plumes of duration $2 \mathrm{~h}$, and the dashed lines for $10 \mathrm{~h}$.

However, at levels close to the level of identification, quantitative estimates will be of great uncertainty and only qualitative identification is possible.

Assuming a well-defined background, the uncertainty in $S_{1}$ and $S_{2}$ due to the Poisson counting statistics will be:

$$
\sigma\left(S_{1}\right)=\sqrt{S_{1}+480}
$$




$$
\sigma\left(S_{2}\right)=\sqrt{S_{2}+960}
$$

We have modeled uncertainty propagation, assuming Poisson distribution of the total number of counts in the ROI for the corresponding time interval. The results are illustrated in Figure 10.

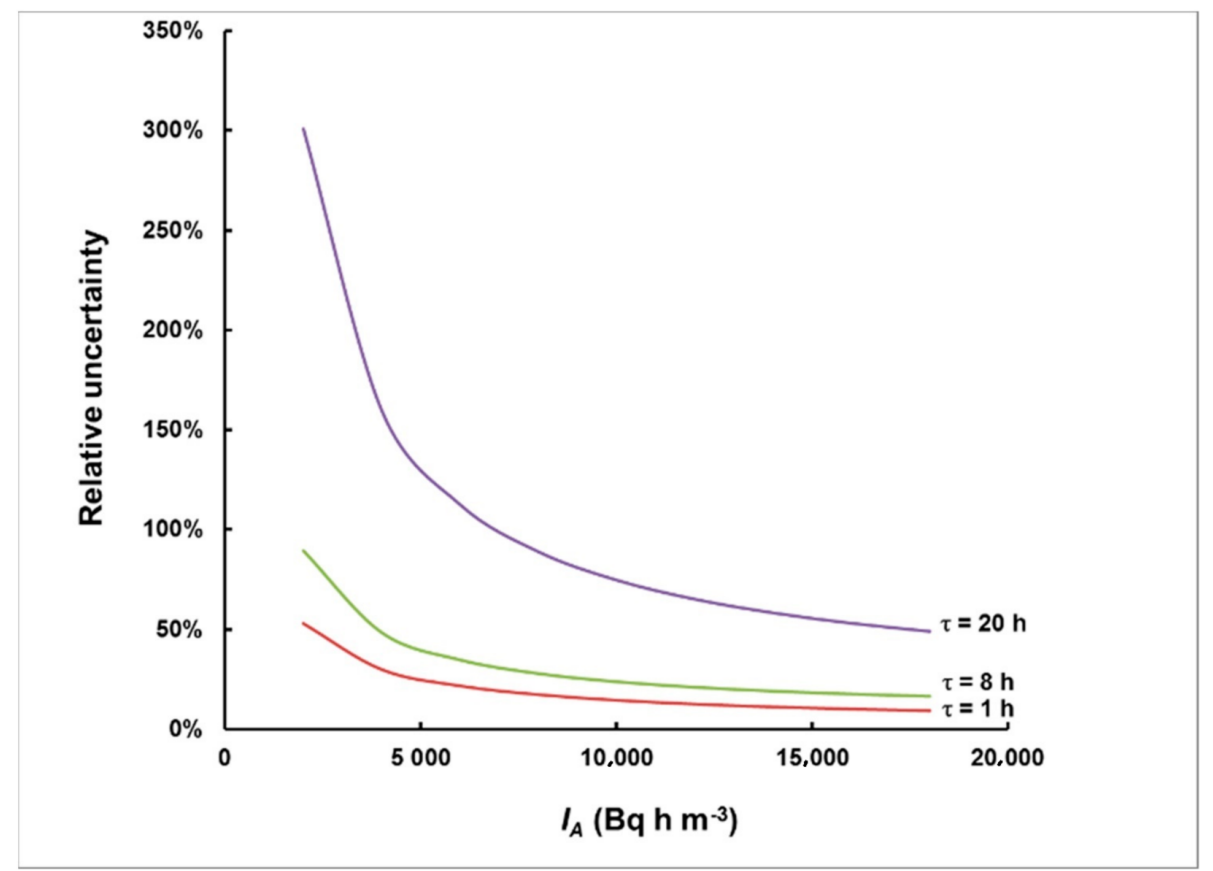

Figure 10. Relative uncertainty in the integrated activity concentration for measurements started with a delay of $1 \mathrm{~h}, 8 \mathrm{~h}$ and $20 \mathrm{~h}$.

As can be seen in Figure 10, the relative uncertainty is substantially influenced by the delay time. For a delay time of $20 \mathrm{~h}$, only levels greater than $20,000 \mathrm{~Bq} \mathrm{~h} \mathrm{~m} \mathrm{~m}^{-3}$ can be measured within $50 \%$ relative uncertainty, while, for a delay time of $8 \mathrm{~h}, 50 \%$ relative uncertainty could be achieved even for levels of approximately $5000 \mathrm{~Bq} \mathrm{~h} \mathrm{~m}$. The delay time of $1 \mathrm{~h}$ makes it possible for levels $>10,000 \mathrm{~Bq} \mathrm{~h} \mathrm{~m}^{-3}$ to be evaluated with a relative uncertainty better than $10 \%$.

\subsection{Simulation of Plumes for an Arbitrary Shape}

To check whether the method may work reliably for pulses with irregular shape, we simulated different plume profiles, illustrated in Figure 11.
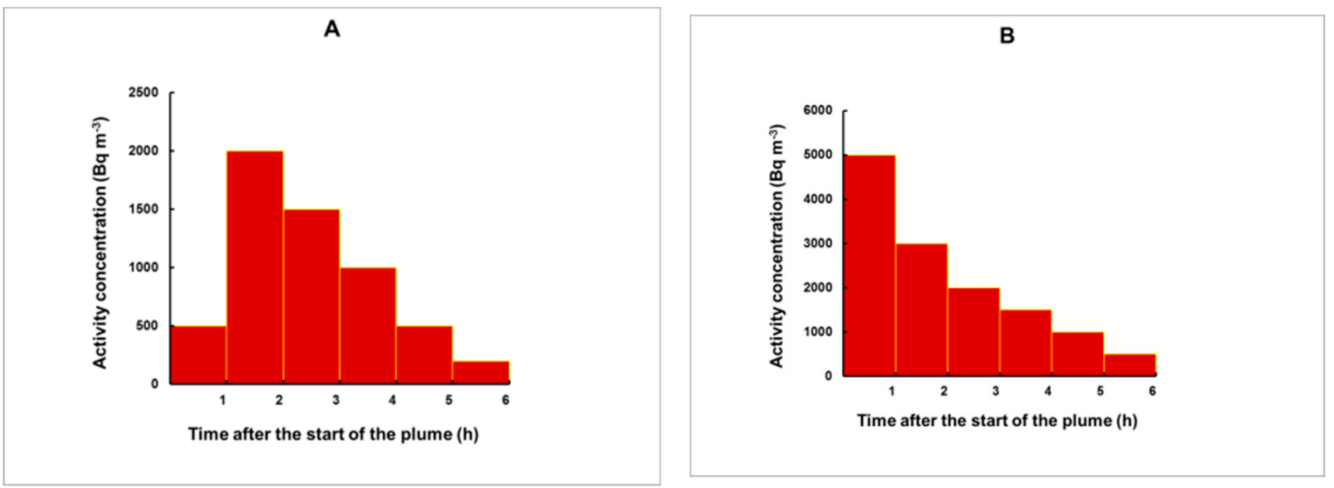

Figure 11. Cont. 

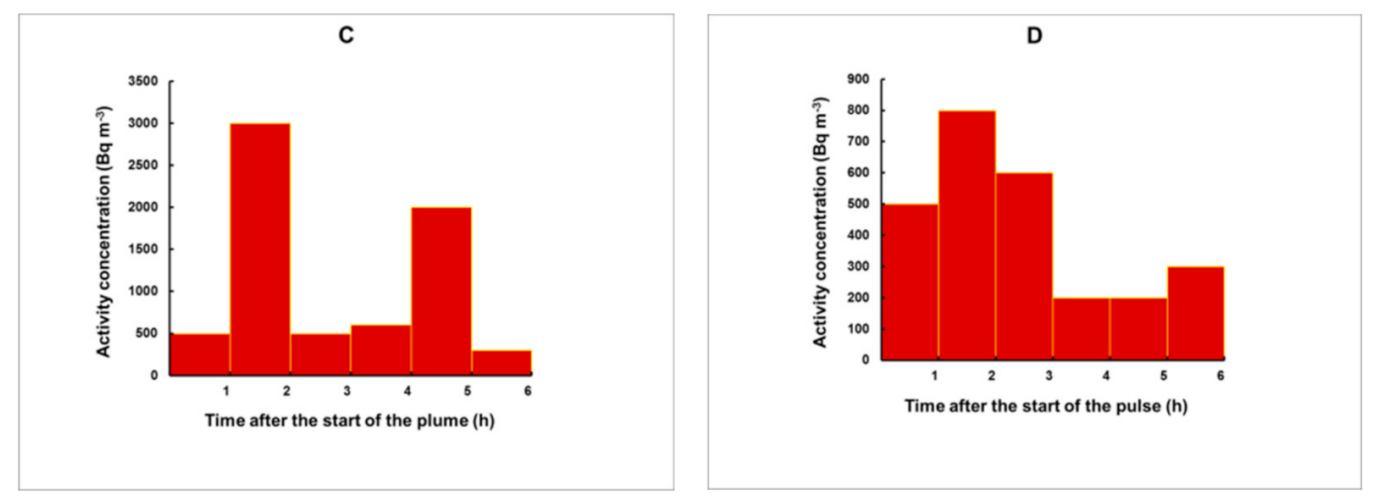

$E$

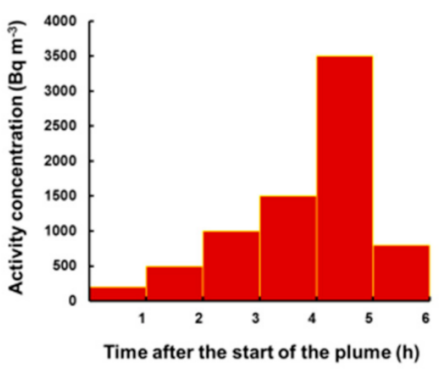

Figure 11. Five different plumes used to test the method. The plumes are composed of consecutive rectangular pulses, each with a duration of $1 \mathrm{~h}$. Different profiles were modelled: Fast (A) and sharp (B) increase followed by a slow decrease; profile with two pulses of higher release (C); relatively weak plume with a release mostly during the first half of the plume (D); a plume with slowly increasing concentrations with a maximum close to its end (E).

With each plume, two "measurement delay scenarios" were considered: the first one commencing $2 \mathrm{~h}$ after the end of the plume and a second one commencing $8 \mathrm{~h}$ after the end of the plume. Because the plumes were of different shape, the delays from the plume's center were different. The delay was calculated by Equation (15) and the integrated activity by Equation (14). The results are illustrated in Figures 12 and 13.

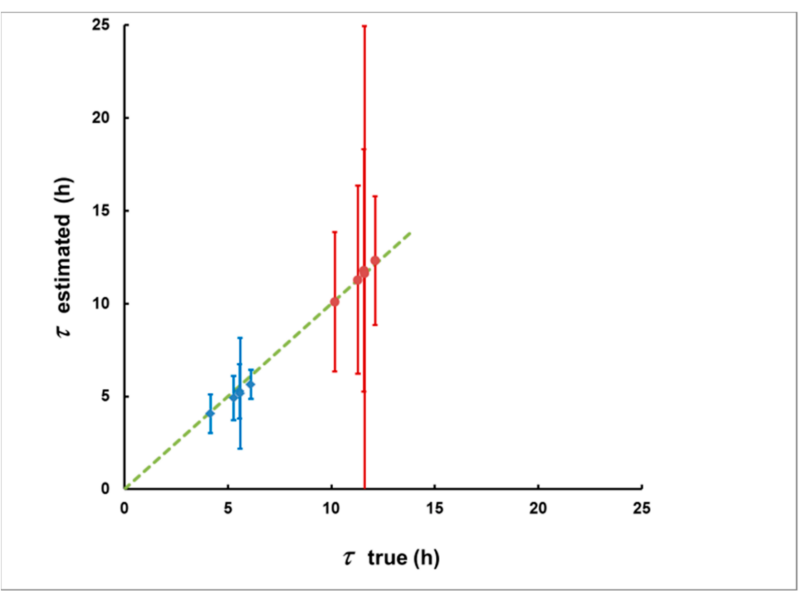

Figure 12. The estimated delays and their uncertainties due to the "counting statistics" (error bars) for the five plumes from Figure 11 and with the two delay scenarios (the first is in blue, the second is in red). Notably, the methodological bias (the deviation of the points from the dashed line that corresponds to the coincidence of the estimated and true delay) is much smaller than the statistical uncertainty. 

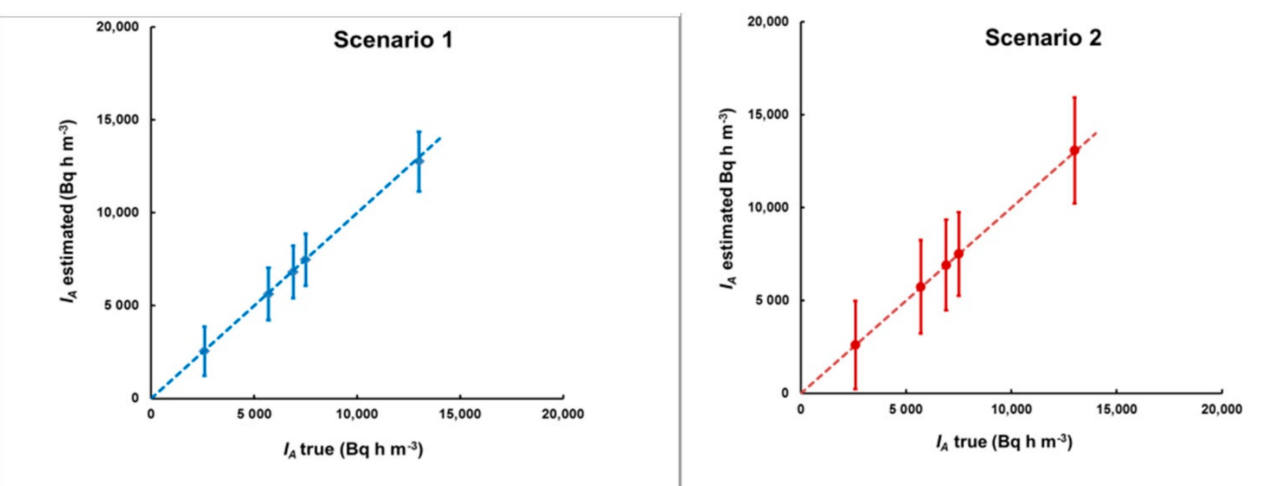

Figure 13. The estimated integrated activity concentrations for the plumes of Figure 11 for the first (left) and the second (right) delay scenario. As with the delay estimates, here, the methodological bias (the deviation of the points from the dashed line that corresponds to "estimated = true $I_{A}$ ") is also much smaller than the "counting statistics uncertainty" (error bars).

As can be seen from Figures 12 and 13, in this case, the method also provides acceptable agreement between the estimated and "true" values. Therefore, we may conclude that the method can be applied for plumes of any shape.

\section{Discussion and Conclusions}

The present work demonstrates, by computer modeling, that, in polycarbonate specimens composed of Makrofol $\mathrm{N}$ foils of thickness $40 \mu \mathrm{m}$ and $120 \mu \mathrm{m}$, exposed for a certain time interval in which a short-term ${ }^{133}$ Xe plume occurs, and with consecutive HPGe gamma spectrometry measurement in two time intervals, the following plume characteristics can be determined:

- The time (before the start of the gamma spectrometry measurement) at which the center of the plume was situated;

- The ${ }^{133}$ Xe activity concentration, integrated over the total duration of the plume, provided that the plume ended before the specimen was taken from the place of exposure. Otherwise, $I_{A}$ will refer to the time from the start of the plume until the moment when the specimen was removed for analysis.

By computer simulation of real plumes of rectangular and arbitrary shape, it was demonstrated (Figure 9) that even plumes of $I_{A}<500 \mathrm{~Bq} \mathrm{~h} \mathrm{~m}^{-3}$ may be identified at $95 \%$ statistical significance if the measurement starts within the first $8 \mathrm{~h}$ after the moment of the plume center. Notably, this estimate was achieved for a gamma spectrometer of average class. The current state-of-the-art in the field of gamma spectrometry includes detectors of much better efficiency and detection systems with better suppressed background than these for the instrument whose characteristics were used. Therefore, one may anticipate that this method has the potential for better sensitivity than that estimated in the current study. Moreover, the choice of foil thickness and counting intervals in the present work was arbitrary, aiming to evaluate the feasibility of the method. Further research may model different foils and counting intervals, aiming for the optimization of the method.

The method provides data that can be useful for the evaluation of other characteristics of the release, e.g., the total released activity. For instance, if the specimen is placed in the ventilation stack through which the activity is released, to obtain the total released activity, one simply has to multiply the determined integrated activity concentration by the air flow rate through the stack. If the specimen is exposed to the environment, the use of an atmospheric transport model along with the data from the measurement will be needed. Thus far, estimates of the released activity, especially after accidents, are associated with great uncertainties and, sometimes, only the order of magnitude of the release may be assessed. In contrast, the modeling revealed that this method may provide $I_{A}$ estimates of uncertainty that may be potentially less than $10 \%$ (see Figure 10). Therefore, the proposed method has the potential to facilitate a step forward in this direction. 
The absorption ability of polycarbonates is not affected by most of environmental factors (pressure, fume, dust, humidity within $0-100 \%$ and even wetting of the material $[33,34])$. The temperature is the only environmental factor of influence, which, however, may be taken into account, provided that the temperature dependences of $K$ and $D$ for xenon in Makrofol N are well-known. Currently, there are data available only for room temperature $\left(22^{\circ} \mathrm{C}\right)$ and the current modeling is based on these data. Dedicated research to complement the data set with values for other temperatures is planned for the future.

One important challenge is to test the method experimentally. Experimental tests with ${ }^{133} \mathrm{Xe}$ are hampered by the difficult and expensive access to certified ${ }^{133} \mathrm{Xe}$ sources and the difficulty of creating a reference ${ }^{133} \mathrm{Xe}$ concentration that can be precisely controlled. We plan in the future to perform such experiments using, as a surrogate of ${ }^{133} \mathrm{Xe}$, the isotope ${ }^{222} \mathrm{Rn}$, for which such tests will be much easier. Of course, this will need revision of the method to incorporate data related to ${ }^{222} \mathrm{Rn}$ for the parameters used.

The polycarbonate material is cheap and offers a cost-efficient option to place many specimens for a long time at different locations in the environment, as well as within a controlled facility, and to use them on occasion, or periodically for regular control. Such a network of specimens may be useful after real or suspected radioxenon release from a nuclear or radiopharmaceutical facility, or to check for possible clandestine nuclear activity. The measured specimens may provide data that will complement the data from stationary monitors and may be used to refine the atmospheric transport models of the plumes. In addition, after outgazing for 10 days or more, the specimen will be suitable for use again. The analysis of the specimens may be conducted in laboratories that are far from the site of exposure, which may be very useful in the event of a nuclear emergency, where the efforts to clarify the situation become international.

Author Contributions: Conceptualization, D.P.; methodology, D.P.; software, P.S., D.P.; validation, D.P., P.S.; formal analysis, D.P.; investigation, D.P.; data curation, D.P.; writing—original draft preparation, D.P.; writing - review and editing, D.P, P.S. All authors have read and agreed to the published version of the manuscript.

Funding: This research received no external funding.

Institutional Review Board Statement: Not applicable.

Informed Consent Statement: Not applicable.

Data Availability Statement: The study did not report any data.

Acknowledgments: The authors are grateful to Tatiana Boshkova for the consultancy in HPGe gamma spectrometry, to Violina Georgieva-Duparinova for the help in preparing the illustrations and to Nadejda Gavritova-Stavreva for the help with the English editing.

Conflicts of Interest: The authors declare no conflict of interest.

\section{References}

1. Commission of the European Communities. Commission recommendation of 18 December 2003 on standardised information on radioactive airborne and liquid discharges into the environment from nuclear power reactors and reprocessing plants in normal operation (notified under document No: C(2003) 4832). Off. J. Eur. Union 2004, L2, 36-46.

2. Stohl, A.; Seibert, P.; Wotawa, G.; Arnold, D.; Burkhart, J.F.; Eckhardt, S.; Tapia, C.; Vargas, A.; Yasunari, T.J. Xenon-133 and caesium-137 releases into the atmosphere from the Fukushima Dai-ichi nuclear power plant: Determination of the source term, atmospheric dispersion, and deposition. Atmos. Chem. Phys. 2012, 12, 2313-2343. [CrossRef]

3. Comprehensive Nuclear-Test-Ban Treaty Organization. Available online: https:/ / www.ctbto.org (accessed on 15 September 2021).

4. Stocki, T.J.; Armand, P.; Heinrich, P.; Ungar, R.K.; D'Amours, R.; Korpach, E.P.; Bellivier, A.; Taffary, T.; Malo, A.; Bean, M.; et al. Measurement and modelling of radioxenon plumes in the Ottawa Valley. J. Environ. Radioact. 2008, 99, 1775-1788. [CrossRef] [PubMed]

5. Mitev, K.; Cassette, P. Radioactive noble gas detection and measurement with plastic scintillators. In Plastic Scintillators; Hamel, M., Ed.; Springer: Basel, Switzerland, 2021; pp. 385-423.

6. Kalinowski, M.B.; Tatlisu, H. Global Radioxenon Emission Inventory from Nuclear Power Plants for the Calendar Year. Pure Appl. Geophys. 2014, 178, 2695-2708. [CrossRef] 
7. Saey, P.R.J.; Bean, M.; Becker, A.; Coyne, J.; D’Amours, R.; De Geer, L.-E.; Hogue, R.; Stocki, T.J.; Ungar, R.K.; Wotawa, G. A long distance measurement of radioxenon in Yellowknife, Canada, in late October 2006. Geophys. Res. Lett. 2007, 34, L20802. [CrossRef]

8. International Atomic Energy Agency. Chernobyl's Legacy: Health, Environmental and Socio-Economic Impacts and Recommendations to the Governments of Belarus, the Russian Federation and Ukraine; Kinly, D., III, Ed.; IAEA: Vienna, Austria, 2005.

9. Stohl, A.; Seibert, P.; Wotawa, G. The total release of xenon-133 from the Fukushima Dai-ichi nuclear power plant accident. J. Environ. Radioact. 2012, 112, 155-159. [CrossRef]

10. Perkins, R.W.; Casey, L.A. Radioxenons: Their Role in Monitoring a Comprehensive Test Ban Treaty; Rep. DOE/RL-96-1; Pac. Northwest Natl. Lab.: Richland, WA, USA, 1996.

11. Leith, W. Geologic and Engineering Constraints on the Feasibility of Clandestine Nuclear Testing by Decoupling in Large Underground Cavities; U.S. Geol. Surv. Open File Rep.: Reston, Virginia, 2001; pp. 1-28.

12. Bowyer, T.W.; Biegalski, S.R.; Cooper, M.; Eslinger, P.W.; Haas, D.; Hayes, J.C.; Miley, H.S.; Strom, D.J.; Woods, V. Elevated radioxenon detected remotely following the Fukushima nuclear accident. J. Environ. Radioact. 2011, 102, 681-687. [CrossRef]

13. Sinclair, L.E.; Seywerd, H.C.J.; Fortin, R.; Carson, J.M.; Saull, P.R.B.; Coyle, M.J.; Van Brabant, R.A.; Buckle, J.L.; Desjardins, S.M.; Hall, R.M. Aerial measurement of radioxenon concentration off the west coast of Vancouver Island following the Fukushima reactor accident. J. Environ. Radioact. 2011, 102, 1018-1023. [CrossRef]

14. Möre, H.; Hubbard, L.M. ${ }^{222}$ Rn absorption in plastic holders for alpha track detectors: A source of error. Radiat. Prot. Dosim. 1997, 74, 85-91. [CrossRef]

15. Pressyanov, D.; Van Deynse, A.; Buysse, J.; Poffijn, A.; Meesen, G. Polycarbonates: A new retrospective radon monitor. In Proceedings of the IRPA Regional Congress on Radiation Protection in Central Europe, Budapest, Hungary, 23-27 August 1999; pp. 716-722.

16. Pressyanov, D.S.; Mitev, K.K.; Stefanov, V.H. Measurements of ${ }^{85} \mathrm{Kr}$ and ${ }^{133}$ Xe by absorption in Makrofol. Nucl. Instrum. Methods Phys. Res. 2004, 527, 657-659. [CrossRef]

17. Pressyanov, D. Modeling a ${ }^{222} \mathrm{Rn}$ measurement technique based on absorption in polycarbonates and track-etch counting. Health Phys. 2009, 97, 604-612. [CrossRef]

18. Pressyanov, D.; Mitev, K.; Georgiev, S.; Dimitrova, I. Sorption and desorption of radioactive noble gases in polycarbonates. Nucl. Instrum. Methods Phys. Res. 2009, 598, 620-627. [CrossRef]

19. Georgiev, S.; Mitev, K.; Pressyanov, D.; Boshkova, T.; Dimitrova, I. Measurement of Xe-133 in Air by Absorption in PolycarbonatesDetection Limits and Potential Applications. In Proceedings of the 2011 IEEE Nuclear Science Symposium Conference Record NP1.M-85, Valencia, Spain, 23-29 October 2011.

20. Mitev, K.; Zhivkova, V.; Pressyanov, D.; Georgiev, S.; Dimitrova, I.; Gerganov, G.; Boshkova, T.; Pressyanov, D. Liquid scintillation counting of polycarbonates: A sensitive technique for measurement of activity concentration of some radioactive noble gases. Appl. Radiat. Isot. 2014, 93, 87-95. [CrossRef]

21. Pressyanov, D.; Mitev, K.; Dimitrova, I.; Georgiev, S. Solubility of krypton, xenon and radon in polycarbonates. Application for measurement of their radioactive isotopes. Nucl. Instrum. Methods Phys. Res. 2011, 629, 323-328. [CrossRef]

22. Tommasino, L.; Tommasino, M.C.; Viola, P. Radon-film-badges by solid radiators to complement track detector-based radon monitors. Radiat. Meas. 2009, 44, 719-723. [CrossRef]

23. Mitev, K.; Cassette, P.; Georgiev, S.; Dimitrova, I.; Sabot, B.; Boshkova, T.; Tartès, I.; Pressyanov, D. Determination of ${ }^{222}$ Rn absorption properties of polycarbonate foils by liquid scintillation counting. Application to ${ }^{222} \mathrm{Rn}$ measurements. Appl. Radiat. Isot. 2016, 109, 270-275. [CrossRef]

24. Mitev, K.; Cassette, P.; Tartès, I.; Georgiev, S.; Dimitrova, I.; Pressyanov, D. Diffusion lengths and partition coefficients of $131 \mathrm{~m}$ Xe and ${ }^{85} \mathrm{Kr}$ in Makrofol N and Makrofol DE polycarbonates. Appl. Radiat. Isot. 2018, 134, 269-274. [CrossRef]

25. Laboratoire National Henri Becquerel. Available online: https://lnhb.br/donnees-nucleaires/donnees-nucleaires-tableau (accessed on 16 November 2021).

26. Tommasino, L. Radon film-badges versus existing passive monitors based on track etch detectors. Nukleonika 2010, 55, 549-553.

27. Tommasino, L.; Tomasino, M.C.; Espinosa, G. Radon film badges based on radon-sorption in solids. A new field for solving long-lasting problems. Rev. Mex. Fis. 2010, S56, 1-4.

28. Pressyanov, D. Modeling response of radon track detectors with solid absorbers as radiators. Radiat. Meas. 2011, 46, 357-361. [CrossRef]

29. Pressyanov, D.; Georgiev, S.; Dimitrova, I.; Mitev, K. Experimental study of the response of radon track detectors with solid absorbers as radiators. Radiat. Meas. 2013, 50, 141-144. [CrossRef]

30. Pressyanov, D.; Georgiev, S.; Dimitrova, I.; Mitev, K.; Boshkova, T. Determination of the diffusion coefficient and solubility of radon in plastics. Radiat. Prot. Dosim. 2011, 145, 123-126. [CrossRef]

31. Taylor, B.N.; Kuyatt, C.E. Guidelines for Evaluating and Expressing the Uncertainty of NIST Measurement Results. In NIST Technical Note 1297; 1994. Available online: https: / / emtoolbox.nist.gov/Publications/NISTTechnicalNote1297s.pdf (accessed on 20 September 2021).

32. Currie, L.A. Limits for Qualitative Detection and Quantitative Determination. Anal. Chem. 1968, 40, 586-593. [CrossRef] 
33. Pressyanov, D.; Buysse, J.; Poffijn, A.; Van Deynse, A.; Meesen, G. Integrated measurements of ${ }^{222}$ Rn by absorption in Makrofol. Nucl. Instrum. Methods Phys. Res. 2004, 516, 203-208. [CrossRef]

34. Dimitrov, D.; Pressyanov, D. The CD/DVD method as a tool for the health physics service and ventilation diagnostics in underground mines. Radiat. Prot. Dosim. 2018, 181, 30-33. [CrossRef] 